\title{
Simulation Research of Micro-atomizer Flat-plate Dielectric-barrier
}

\section{Discharge}

\author{
Xia Xuwei ${ }^{1, \mathrm{a}}$, Zhou Binbin ${ }^{2, \mathrm{~b}}$, Fu Hao ${ }^{2, \mathrm{c}}$, Yuan Hailing ${ }^{2, \mathrm{~d}}$, Liao Honghua,e
}

1. Chongqing special equipment inspection and research institute, Northern New Area, China, 401121;

2. School of Information and Engineering, Hubei University for Nationalities, Enshi, China, 445000

a41549003@qq.com, b245594560@qq.com, '979680779@qq.com, d418980226@qq.com, e405219300@qq.com

Keywords: Ansys; Micro-atomizer; Flat-plate type dielectric barrier discharge; Discharge gap; Density of discharge.

Abstract: A flat-plate type dielectric barrier discharge of micro-atomizer was studied based on Ansys software in this paper. And it was mainly studied the effect of the discharge characteristics on the different factors, such as the material and thickness of dielectric barrier, the discharge gap, the shape of exciting electrodes, the exciting voltage, and so on. The simulation results show that if the greater dielectric constant of dielectric barrier material was choose, and the larger discharge density would be easy to obtain. To choose the round exciting electrodes can be get more discharge density than the square exciting electrodes. Correspondingly, to select the thinner dielectric barrier material can be easy to obtain the more discharge density, and to design the smaller discharge gap can be propitious to enhance the discharge density and improve the uniformity of discharge, and so on.

\section{Introduction}

Dielectric Barrier Discharge is short for DBD. It is a kind of the low temperature plasma what can maintain it under atmospheric pressure ${ }^{[1]}$. Compared with the other types of plasma, DBD has a good ability of cracking and inspire. It can be operated under a variety of gases, It has some features, such as simple structure, small volume, low power consumption, low operation temperature, generated naturally, ect ${ }^{[2-3]}$. Application of DBD micro plasma in hydride generation, Development of $\mathrm{DBD}$ in low temperature miniature atomization device, it will be very conducive to the miniaturization of atomic spectroscopy ${ }^{[4-5]}$.

According to the needs of the project, a flat-plate type dielectric barrier discharge of low temperature micro-atomizer was simulation and studied, and it was mainly studied the effect of the discharge characteristics on the different factor, such as different dielectric barrier material, the thickness of the different medium, different discharge gap and electrode shape and different incentives voltage ${ }^{[6-8]}$. These conclusions based on the DBD has lay the theoretical foundation for the the further research and design of the low temperature miniature atomization device .

\section{The type of flat-plate DBD structure and equivalent circuit model}

The flat-plate miniature atomization for DBD in the low temperatur are made up of high-voltage excitation signal source, incentive electrode, discharge chamber, DBD layer, gas inlet and outlet channel, and so on. As shown in figure 1(a), the flat-plate DBD discharge in air gap are 
made up of random distribution of micro discharge in time and space ${ }^{[9]}$. The equivalent circuit of DBD is different before and after micro discharge. DBD equivalent circuit is shown in Figure 1(b) before discharge. Micro discharge has not occurred, the whole circuit is divided into the blocking medium and the air gap. The equivalent circuit of DBD is composed of dielectric equivalent capacitance $\mathrm{Cd}$ and air gap equivalent capacitance $\mathrm{Cg}$ series. After the air gap breakdown, micro discharge occurs, Ionization of air gap is in different levels that caused the nonlinear change in $\mathrm{Cg}$. At this time, The equivalent circuit of DBD is composed of $\mathrm{Cd}$ and micro discharge equivalent circuit, as shown in Figure 1(c).

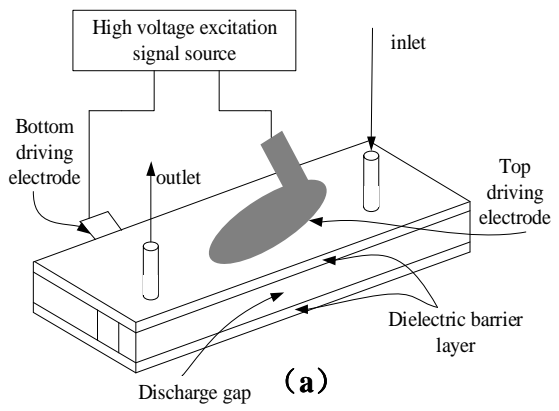

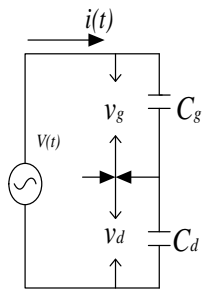

(b)

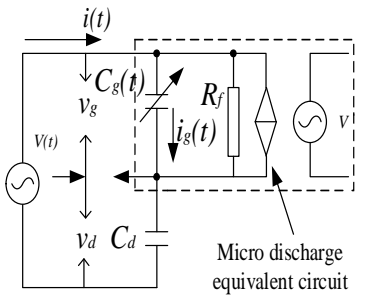

(c)

Fig. 1 The structural diagram and equivalent circuit model of flat-plate DBD

\section{DBD modeling based on Ansys}

When using the Ansys software simulation, the process of Ansys operation is as follow: (1) Start the Solidworks software and set up the working file name and title. (2) Draw the simulation model and cooperate with it. (3) Define material properties. (4) Save model. (5) Attribute to the established model and divide the mesh. (6) Applying loading solution. (7) View the results and other steps.

When using the Ansys software simulation, medium material is choose as epoxy resin, glass and PFTE, respectively. The dielectric constant of them are 3 4, 4.1 and $2.55 \mathrm{~F} / \mathrm{m}$, respectively. Their shapes are $50 \mathrm{~mm} \times 50 \mathrm{~mm}$ square. When studying the effect of different electrode shapes on DBD, $32 \mathrm{~mm} \times 32 \mathrm{~mm}$ square was used for the excitation electrode, choose on convex octagon's square of $32 \mathrm{~mm} \times 32 \mathrm{~mm}$ and cut corners to $5 \mathrm{~mm}$, the circle of copper electrode is $32 \mathrm{~mm}$. When studying the influence of different thickness of medium on DBD, the thickness of the glass is selected as 1, 2 and $3 \mathrm{~mm}$. When studying the influence of different discharge gap on DBD, the discharge gap between the glass plate and the plate is 1,2 and $3 \mathrm{~mm}$. When studying the effect of different excitation voltage on $\mathrm{DBD}$, the excitation voltage is set as $1,3,6,11,15,19 \mathrm{kV}$, respectively.

\section{Simulation results are analyzed and discussed}

\section{Effects of different dielectric materials on discharge characteristics}

Epoxy resin, glass and Teflon (PTFE) were used to study the influence of different materials on the DBD. The Shape of square are $50 \mathrm{~mm} \times 50 \mathrm{~mm}$. The thickness is $1 \mathrm{~mm}$. The discharge gap between the plate and the plate is $2 \mathrm{~mm}$. When the $10 \mathrm{KV}$ voltage is applied between the upper electrode and the lower electrode, the discharge unit current density of different dielectric materials is shown in Figure 2, Figure 2 (a), (b), (c) are epoxy resin, glass, and PTFE, respectively. 


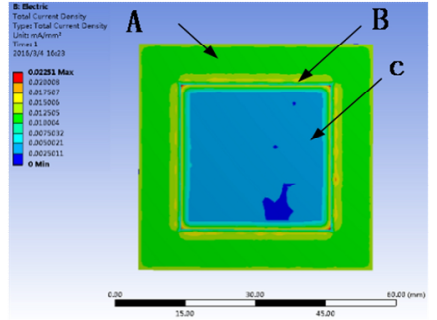

(a)

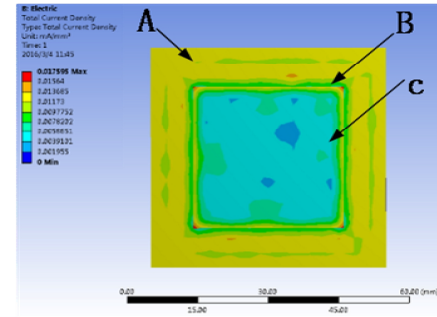

(b)

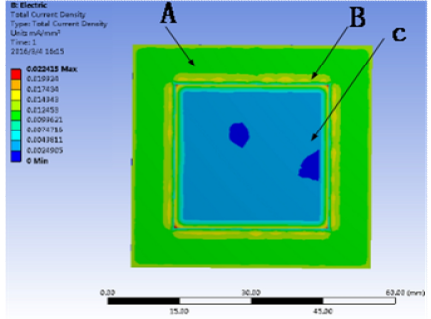

(c)

Fig.2. The discharge unit current density with different dielectric materia

It is obvious from Figure 2, the surface area of DBD is green in Figure (a), the unit of current density is bettwen from $0.010004 \mathrm{~mA} / \mathrm{mm}^{2}$ to $0.012505 \mathrm{~mA} / \mathrm{mm}^{2}$, the edge B area of incentive electrode is yellow, the unit of current density is bettwen from $0.015006 \mathrm{~mA} / \mathrm{mm}^{2}$ to $0.017507 \mathrm{~mA} / \mathrm{mm}^{2}$, the surface of the electrode $\mathrm{C}$ area is indigo, the unit of current density is bettwen from $0.0025011 \mathrm{~mA} / \mathrm{mm}^{2}$ to $0.0050021 \mathrm{~mA} / \mathrm{mm}^{2}$. The surface area of DBD is yewlow in Figure(b), the unit of current density is bettwen from $0.01173 \mathrm{~mA} / \mathrm{mm}^{2}$ to $0.013685 \mathrm{~mA} / \mathrm{mm}^{2}$, the edge $\mathrm{B}$ area of incentive electrode is green, the unit of current density is bettwen from $0.0078202 \mathrm{~mA} / \mathrm{mm}^{2}$ to $0.0097752 \mathrm{~mA} / \mathrm{mm}^{2}$, the edge $\mathrm{C}$ area of incentive electrode is blue, the unit of current density is bettwen from $0.0039101 \mathrm{~mA} / \mathrm{mm}^{2}$ to $0.0058651 \mathrm{~mA} / \mathrm{mm}^{2}$. The surface area of DBD is green in Figure(c), the unit of current density is bettwen from $0.0099621 \mathrm{~mA} / \mathrm{mm}^{2}$ to $0.012453 \mathrm{~mA} / \mathrm{mm}^{2}$, the edge $\mathrm{B}$ area of incentive electrode is green, the unit of current density is bettwen from $0.014943 \mathrm{~mA} / \mathrm{mm}^{2}$ to $0.017434 \mathrm{~mA} / \mathrm{mm}^{2}$, the edge $\mathrm{C}$ area of incentive electrode is indigo, the unit of current density is bettwen from $0.0024905 \mathrm{~mA} / \mathrm{mm}^{2}$ to $0.0049811 \mathrm{~mA} / \mathrm{mm}^{2}$. Comparative analysis of Figure 2 (a), (b), (c) result showed that the selection of glass surface of the medium showed yellow level area is much larger than the selection of epoxy resin, PTFE dielectric surface presented green regional level, the conclusion shows that the bigger relative dielectric constant can of easier get DBD.

\section{Different shapes of electrode on effect on discharge characteristics}

The excitation electrode has choose as square of $31 \mathrm{~mm}$ x $31 \mathrm{~mm}$, choose on convex octagon's square of $31 \mathrm{~mm} \times 31 \mathrm{~mm}$ and cut corners to $6 \mathrm{~mm}$, the diameter of the circular copper electrode is $32 \mathrm{~mm}$, material of DBD has choose as glass, the thickness of copper electrode and glass are all set to $1 \mathrm{~mm}$, the discharge gap between the plate and the plate is $2 \mathrm{~mm}$. When the $10 \mathrm{KV}$ voltage is applied between the top electrode and the bottom electrode, the discharge unit current density of different shapes of incentive electrode is shown in Figure3, and Figure3(a), (b), (c) are shown in the shape of square, convex octagon, circular, respectively.

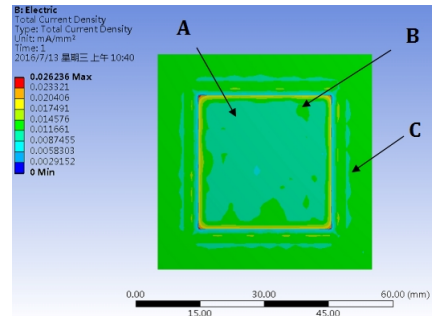

(a)

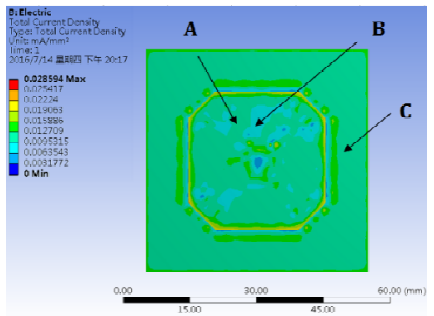

(b)

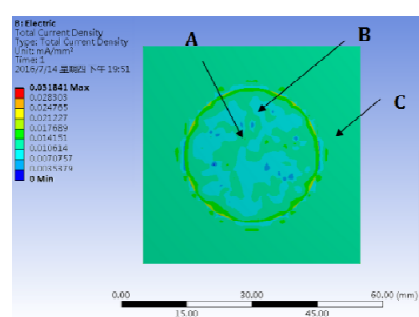

(c)

Fig.3. The discharge unit current density with different exciting electrode shapes

It is obvious from Figure 3, the surface area of DBD is turquoise in Figure (a), the unit of current density is bettwen from $0.0058303 \mathrm{~mA} / \mathrm{mm}^{2}$ to $0.0087455 \mathrm{~mA} / \mathrm{mm}^{2}$, the edge $\mathrm{B}$ area of incentive electrode is green, the unit of current density is bettwen from $0.011661 \mathrm{~mA} / \mathrm{mm}^{2}$ to $0.014576 \mathrm{~mA} / \mathrm{mm}^{2}$, the surface of the electrode $\mathrm{C}$ area is green, the unit of current density is bettwen from $0.011661 \mathrm{~mA} / \mathrm{mm}^{2}$ to $0.014576 \mathrm{~mA} / \mathrm{mm}^{2}$. The surface area of DBD is turquoise in Figure (b), 
the unit of current density is bettwen from $0.0095315 \mathrm{~mA} / \mathrm{mm}^{2}$ to $0.012709 \mathrm{~mA} / \mathrm{mm}^{2}$, the edge B area of incentive electrode is blue, the unit of current density is bettwen from $0.0063543 \mathrm{~mA} / \mathrm{mm}^{2}$ to $0.0095315 \mathrm{~mA} / \mathrm{mm}^{2}$, the surface of the electrode $\mathrm{C}$ area is turquoise, the unit of current density is bettwen from $0.0095315 \mathrm{~mA} / \mathrm{mm}^{2}$ to $0.012709 \mathrm{~mA} / \mathrm{mm}^{2}$. The surface area of DBD is turquoise in Figure(c), the unit of current density is bettwen from $0.010614 \mathrm{~mA} / \mathrm{mm}^{2}$ to $0.014151 \mathrm{~mA} / \mathrm{mm}^{2}$, the edge $\mathrm{B}$ area of incentive electrode is blue, the unit of current density is bettwen from $0.0070757 \mathrm{~mA} / \mathrm{mm}^{2}$ to $0.010614 \mathrm{~mA} / \mathrm{mm}^{2}$, the edge $\mathrm{C}$ area of incentive electrode is turquoise, the unit of current density is bettwen from $0.010614 \mathrm{~mA} / \mathrm{mm}^{2}$ to $0.014151 \mathrm{~mA} / \mathrm{mm}^{2}$.Comparison of the Figure3 (a),(b),(c) shows that under the condition, the use of circular excitation electrode to obtain the discharge density is far greater than the use of square excitation electrodes and convex.

\section{effects of different dielectric thickness on discharge characteristics}

The $50 \mathrm{~mm} \times 50 \mathrm{~mm}$ square glass is used as the dielectric barrier layer, the thickness of the glass is selected as $1 \mathrm{~mm}, 2 \mathrm{~mm}$ and $3 \mathrm{~mm}$, the discharge gap between the plate and the plate is $2 \mathrm{~mm}$. When the $10 \mathrm{KV}$ voltage is applied between the upper electrode and the lower electrode. The discharge unit current density of different thickness of the glass is shown in Figure 4, Figure 4 (a), (b), (c) are 1,2 and 3mm, respectively.

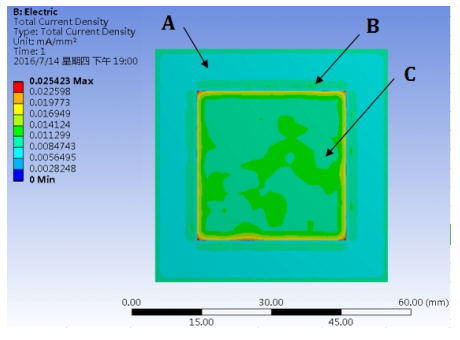

(a)

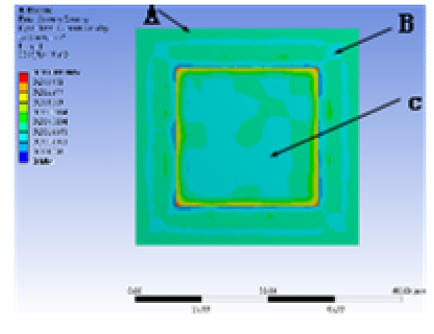

(b)

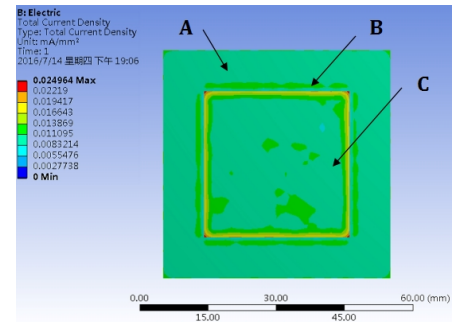

(c)

Fig.4. The discharge unit current density with different thickness of dielectric materials

It is obvious from Figure 4, the surface area of DBD is blue in Figure (a), the unit of current density is bettwen from $0.0056495 \mathrm{~mA} / \mathrm{mm}^{2}$ to $0.0084743 \mathrm{~mA} / \mathrm{mm}^{2}$, the edge $\mathrm{B}$ area of incentive electrode is turquoise, the unit of current density is bettwen from $0.0084743 \mathrm{~mA} / \mathrm{mm}^{2}$ to $0.011299 \mathrm{~mA} / \mathrm{mm}^{2}$, the surface of the electrode $\mathrm{C}$ area is turquoise, the unit of current density is bettwen from $0.0084743 \mathrm{~mA} / \mathrm{mm}^{2}$ to $0.011299 \mathrm{~mA} / \mathrm{mm}^{2}$. The surface area of DBD is turquoise in Figure(b), the unit of current density is bettwen from $0.0059343 \mathrm{~mA} / \mathrm{mm}^{2}$ to $0.0079124 \mathrm{~mA} / \mathrm{mm}^{2}$, the edge $B$ area of incentive electrode is blue, the unit of current density is bettwen from $0.0039562 \mathrm{~mA} / \mathrm{mm}^{2}$ to $0.0059343 \mathrm{~mA} / \mathrm{mm}^{2}$, the surface of the electrode C area is blue, the unit of current density is bettwen from $0.0039562 \mathrm{~mA} / \mathrm{mm}^{2}$ to $0.0059343 \mathrm{~mA} / \mathrm{mm}^{2}$. The surface area of DBD is green in Figure(c), the unit of current density is bettwen from $0.0055476 \mathrm{~mA} / \mathrm{mm}^{2}$ to $0.0083214 \mathrm{~mA} / \mathrm{mm}^{2}$, the edge $\mathrm{B}$ area of incentive electrode is green, the unit of $\mathrm{B}$ area'scurrent density is bettwen from $0.011095 \mathrm{~mA} / \mathrm{mm}^{2}$ to $0.013869 \mathrm{~mA} / \mathrm{mm}^{2}$, the edge $\mathrm{C}$ area of incentive electrode is blue, the unit of current density is bettwen from $0.0055476 \mathrm{~mA} / \mathrm{mm}^{2}$ to $0.0083214 \mathrm{~mA} / \mathrm{mm}^{2}$. Comparison of the Figure4 (a),(b),(c) shows that under the condition, the thinner dielectric barrier material can be easy to obtain the more discharge density.

\section{effect of different discharge gap on discharge characteristics}

The thickness is $1 \mathrm{~mm}$ and $50 \mathrm{~mm} \times 50 \mathrm{~mm}$ square glass are used as the dielectric barrier layer. The discharge gap between the plate and the plate is 1,2 and $3 \mathrm{~mm}$, respectively. When the $10 \mathrm{kV}$ voltage is applied between the upper electrode and the lower electrodethe, discharge unit current density of different discharge gap is shown in Figure 5. Figure 5 (a), (b), (c) are 1,2 and $3 \mathrm{~mm}$,respectively. 


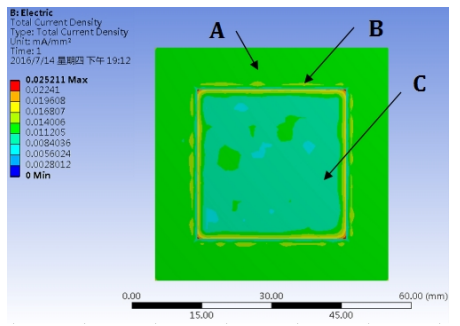

(a)

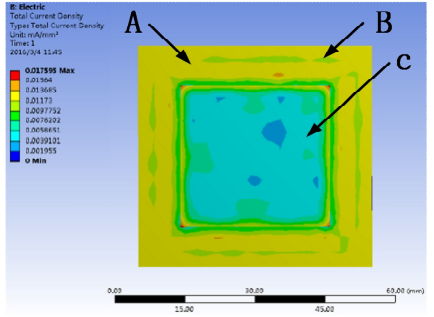

(b)

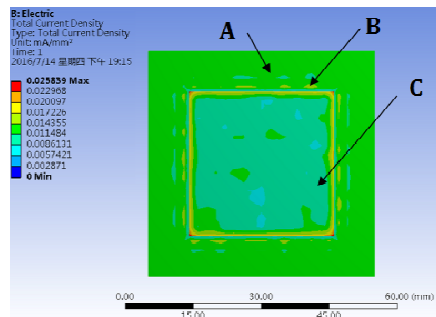

(c)

Fig.5. The discharge unit current density with different discharge gap

It is obvious from Figure 5, the surface area of DBD is green in Figure (a), the unit of current density is bettwen from $0.011205 \mathrm{~mA} / \mathrm{mm}^{2}$ to $0.014006 \mathrm{~mA} / \mathrm{mm}^{2}$, the edge $\mathrm{B}$ area of incentive electrode is yewlow, the unit of current density is bettwen from $0.016807 \mathrm{~mA} / \mathrm{mm}^{2}$ to $0.019608 \mathrm{~mA} / \mathrm{mm}^{2}$, the surface of the electrode $\mathrm{C}$ area is blue, the unit of current density is bettwen from $0.0056024 \mathrm{~mA} / \mathrm{mm}^{2}$ to $0.0084036 \mathrm{~mA} / \mathrm{mm}^{2}$. The surface area of DBD is yewlow in Figure (b), the unit of current density is bettwen from $0.01173 \mathrm{~mA} / \mathrm{mm}^{2}$ to $0.013685 \mathrm{~mA} / \mathrm{mm}^{2}$, the edge B area of incentive electrode is green, the unit of current density is bettwen from $0.0078202 \mathrm{~mA} / \mathrm{mm}^{2}$ to $0.0097752 \mathrm{~mA} / \mathrm{mm}^{2}$, the surface of the electrode $\mathrm{C}$ area is blue, the unit of current density is bettwen from $0.0039101 \mathrm{~mA} / \mathrm{mm}^{2}$ to $0.0058651 \mathrm{~mA} / \mathrm{mm}^{2}$. In Figure (c), the unit of surface area A current density is bettwen from $0.011484 \mathrm{~mA} / \mathrm{mm}^{2}$ to $0.014355 \mathrm{~mA} / \mathrm{mm}^{2}$, the unit of current density B is bettwen from $0.017226 \mathrm{~mA} / \mathrm{mm}^{2}$ to $0.020097 \mathrm{~mA} / \mathrm{mm}^{2}$, the area $\mathrm{C}$ is blue, the unit of current density is bettwen from $0.0057421 \mathrm{~mA} / \mathrm{mm}^{2}$ to $0.0086131 \mathrm{~mA} / \mathrm{mm}^{2}$. Comparative analysis of Figure 5 (a),(b),(c) can be known that unit current density of the glass dielectric of $2 \mathrm{~mm}$ discharge gap is greater than that of $1 \mathrm{~mm}$ or $3 \mathrm{~mm}$ discharge gap, at the same time, the dielectric surface discharge is more uniform, in other words, the smaller discharge gap is more benefit to improving the density and uniformity of discharge.

\section{effects of changing excitation voltage on discharge characteristics}

The thickness is $1 \mathrm{~mm}$ and $50 \mathrm{~mm} \times 50 \mathrm{~mm}$ square glass are used as the dielectric barrier layer, The discharge gap between the plate and the plate is $2 \mathrm{~mm}$. When the $1,3,6,11,15,19 \mathrm{kV}$ voltage is applied between the upper electrode and the lower electrode, the discharge unit current density of different excitation electrode is shown in Figure 6. Figure 6 (a),(b),(c) are 1,3,6,11,15 and 19kV, respectively.

It is obvious from Figure 6, the surface area of DBD is green in Figure (a), the unit of current density $\mathrm{A}$ is bettwen from $0.0011209 \mathrm{~mA} / \mathrm{mm}^{2}$ to $0.0014012 \mathrm{~mA} / \mathrm{mm}^{2}$, the unit of current density B is bettwen from $0.0016814 \mathrm{~mA} / \mathrm{mm}^{2}$ to $0.0019616 \mathrm{~mA} / \mathrm{mm}^{2}$, the surface of the electrode $\mathrm{C}$ area is blue, the unit of current density $C$ is bettwen from $0.00056046 \mathrm{~mA} / \mathrm{mm}^{2}$ to $0.00084069 \mathrm{~mA} / \mathrm{mm}^{2}$. In Figure(b), the unit of current density A is bettwen from $0.0050293 \mathrm{~mA} / \mathrm{mm}^{2}$ to $0.0060352 \mathrm{~mA} / \mathrm{mm}^{2}$, the unit of current density $B$ is bettwen from $0.0056099 \mathrm{~mA} / \mathrm{mm}^{2}$ to $0.0070124 \mathrm{~mA} / \mathrm{mm}^{2}$, the surface of the electrode $\mathrm{C}$ area is blue, the unit of current density $\mathrm{C}$ is bettwen from $0.002805 \mathrm{~mA} / \mathrm{mm}^{2}$ to $0.0042074 \mathrm{~mA} / \mathrm{mm}^{2}$. 


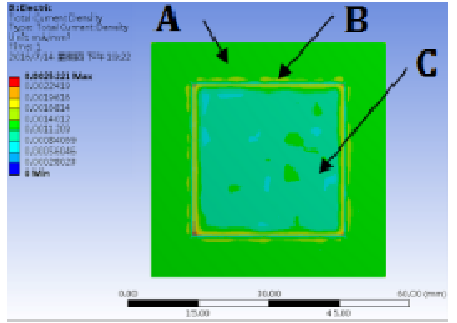

(a)

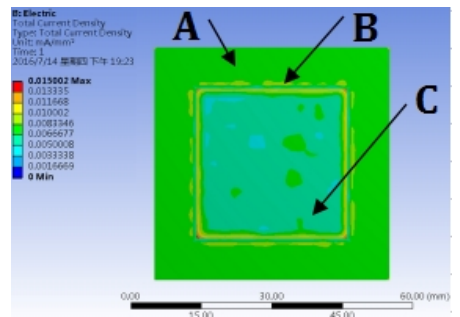

(c)

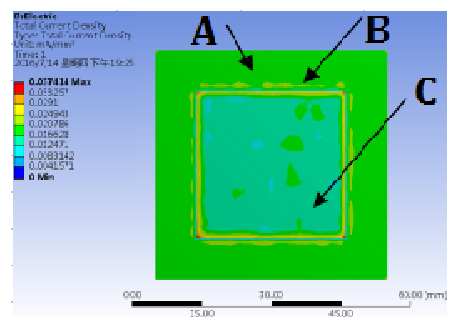

(e)

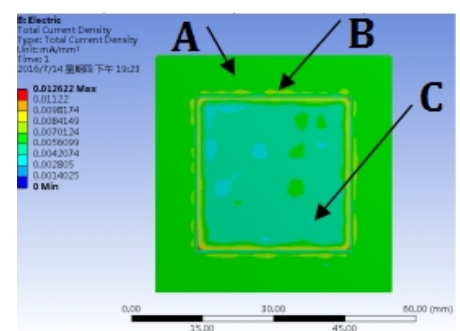

(b)

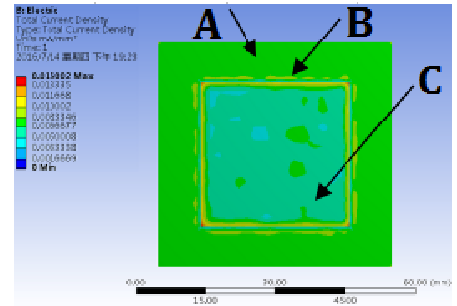

(d)

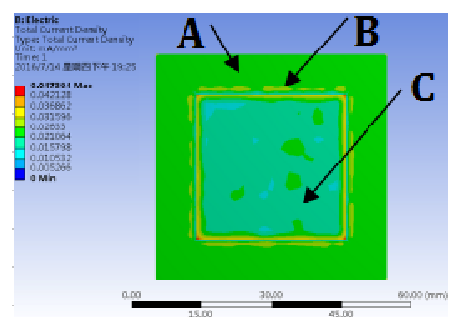

(f)

Fig. 6 The discharge unit current density with different exciting voltage

In Figure (c), the unit of current density $A$ is bettwen from $0.0066677 \mathrm{~mA} / \mathrm{mm}^{2}$ to $0.0083346 \mathrm{~mA} / \mathrm{mm}^{2}$, the unit of current density $\mathrm{B}$ is bettwen from $0.010002 \mathrm{~mA} / \mathrm{mm}^{2}$ to $0.011668 \mathrm{~mA} / \mathrm{mm}^{2}$, the surface of the electrode $\mathrm{C}$ area is blue, the unit of current density $\mathrm{C}$ is bettwen from $0.0033338 \mathrm{~mA} / \mathrm{mm}^{2}$ to $0.0050008 \mathrm{~mA} / \mathrm{mm}^{2}$. In Figure (d), the unit of current density A is bettwen from $0.012531 \mathrm{~mA} / \mathrm{mm}^{2}$ to $0.015663 \mathrm{~mA} / \mathrm{mm}^{2}$, the unit of current density $\mathrm{B}$ is bettwen from $0.018796 \mathrm{~mA} / \mathrm{mm}^{2}$ to $0.021928 \mathrm{~mA} / \mathrm{mm}^{2}$, the surface of the electrode $\mathrm{C}$ area is blue, the unit of current density $\mathrm{C}$ is bettwen from $0.0062653 \mathrm{~mA} / \mathrm{mm}^{2}$ to $0.0093979 \mathrm{~mA} / \mathrm{mm}^{2}$. In Figure (e), the unit of current density $\mathrm{A}$ is bettwen from $0.016628 \mathrm{~mA} / \mathrm{mm}^{2}$ to $0.020786 \mathrm{~mA} / \mathrm{mm}^{2}$, the unit of current density B is bettwen from $0.024943 \mathrm{~mA} / \mathrm{mm}^{2}$ to $0.0291 \mathrm{~mA} / \mathrm{mm}^{2}$, the surface of the electrode $\mathrm{C}$ area is blue, the unit of current density $C$ is bettwen from $0.0083142 \mathrm{~mA} / \mathrm{mm}^{2}$ to $0.012471 \mathrm{~mA} / \mathrm{mm}^{2}$. In Figure (f), the unit of current density $A$ is bettwen from $0.021064 \mathrm{~mA} / \mathrm{mm}^{2}$ to $0.02633 \mathrm{~mA} / \mathrm{mm}^{2}$, the unit of current density B is bettwen from $0.031596 \mathrm{~mA} / \mathrm{mm}^{2}$ to $0.036862 \mathrm{~mA} / \mathrm{mm}^{2}$, the surface of the electrode $C$ area is blue, the unit of current density $C$ is bettwen from $0.010532 \mathrm{~mA} / \mathrm{mm}^{2}$ to $0.015798 \mathrm{~mA} / \mathrm{mm}^{2}$. Comparative analysis of Figure6 (a),(b),(c),(d),(e),(f) can be known that unit current density of larger voltage can get more discharge.

\section{Conclusion}

This paper simulate and analyze the flat-plate type dielectric barrier discharge which is suitable for micro-atomizer by Ansys software, through the analysis of effects of different dielectric barrier materials, different dielectric thickness, different discharge gap, different excitation electrode shapes and different excitation voltage on the discharge characteristics. we come to the conclusion that the larger dielectric constant of dielectric barrier material, the larger unit discharge current density would be easy to obtain. Unit discharge current density obtained from circular excitation electrode is much larger than that of square or convex eight sided shape excitation electrode. 
Relatively speaking, thinner dielectric barrier material is easier to obtain a larger unit discharge current density. Smaller discharge gap is more conducive to improving unit discharge current density and discharge uniformity, and so on. This paper will lay a foundation for the subsequent research and design of micro-atomizer based on dielectric barrier discharge.

\section{Acknowledgements}

This work is supported by National Natural Science Foundation of China (61463014, 61263030), Science Research Program of Eduation Bureau of Hubei Province (T201429), Innovation and Entrepreneurship Training Program for College Students in Hubei Province (201510517010).

\section{References}

[1] Yan Jinyun, Fang Zhi, Qian Chen. Discharge characteristics comparison of dielectric barrier discharge with different electrode structures [J]. Insulating Materials, 2014, 47(5): 110-115.

[2] Li Qingquan, Hao Lingyan. Surface dielectric barrier discharge plasma and its applications [J]. High Voltage Engineering, 2016, 42(4): 1079-1090.

[3] Li Weilan, Cheng Guohong, Zhang Fukuan. Research of catalytic reduction of no by high voltage pulse discharge plasma [J]. Journal of China Three Gorges University(Natural Sciences), 2005, 27(1): 80-82.

[4] Hamed Taghvaei, Mahsa Kheirollahivash, Mohammad Ghasemi, et al. Noncatalytic Upgrading of Anisole in an Atmosphere DBD Plasma Reactor: Effect of Carrier Gas Type, Voltage, and Frequency [J]. Energy \&amp; Fuels, 2014, 28(2): 2535-2543.

[5] Fahad Rehman, Wamesth S. Abdul Majeed, William B. Zimmerman, et al. Hydrogen Production from Water Vapor Plasmolysis Using DBD-Corona Hybrid Reactor [J]. Energy \&amp; Fuels, 2013, 27(3): 2748-2761.

[6] Jiang Ming, Zhou Ping. Theoretical model of non ideal plasma electron density research [J]. Journal of southwest university for nationalities(Natural Science Edition), 2005, 31(6): 116-118.

[7] Florez, David, Diez, et al. Square-Shape Current-Mode Supply for Parametric Control of the DBD Excilamp Power [J]. IEEE Transactions on Industrial Electronics, 2015, 62(3): 1451-1460.

[8] Zhou Zhangwen, Liu Zhan, Sun Dong. Diagnostic dielectric barrier discharge excitation of air density perturbations [J]. Journal of jishou university(Natural Sciences Edition), 2011, 32(5): 62-64-74.

[9] Qi Zewu, Zhang Wei, Li Pinglin, etc. Design of DBD high-frequency high-voltage power Supply and its discharging characteristics [J]. High Voltage Engineering, 2016, 42(3): 807 -812 . 